\title{
Design and Comparison of LEACH and Improved Centralized LEACH in Wireless Sensor Network
}

\author{
Pratiksha Mishra ${ }^{a}$, Satish Kumar Alaria ${ }^{b}$, Prakash Dangic \\ ${ }^{a}$ M.Tech. Scholar, Department of Computer Science \&Engineering, AIET, Jaipur, India \\ ${ }^{b, c}$ Assistant Professor, Department of Computer Science \& Engineering, AIET, Jaipur, India
}

\section{ABSTRACT}

A WSN consists of a setup of sensor nodes/motes which perceives the environment under monitoring, and transfer this information through wireless links to the Base Station (BS) or sink. The sensor nodes can be heterogeneous or homogeneous and can be mobile or stationary. The data gathered is forwarded through single/multiple hops to the BS/sink. In this paper, propose improvements to LEACH routing protocol to reduce energy consumption and extend network life. LEACH Distance Energy (LEACH-DE) not only selects the cluster head node by considering that the remaining energy of the node is greater than the average remaining energy level of the nodes in the network, but also selects the cluster head node parameters based on the geometric distance between the candidate node and the BS. The simulation results show that the algorithm proposed in this work is superior to LEACH and LEACH-C (Centralized) in terms of energy saving and extending the lifetime of wireless sensor networks.

Keywords: WSN, LEACH, LEACH-C, Network Life Time, Base Station, Sensor Node

\section{INTRODUCTION}

There exist significant variances between wired traditional and ad- hoc networks and the WSNs. The distinctions emerge mostly on account of the resource constrains in WSNs related to energy, communication range, bandwidth, processing and memory capabilities. The stringent constraints in resources make it inevitable to have specific design considerations for protocol developments in WSNs. Besides that, the design of WSNs is completely application dependent. The network topology, size of the network and the mode of deployment is based on the application requirement. Because of its unique features and application dependency, the algorithms and protocols used in traditional and ad hoc networks will not suite for WSNs. The major differences among sensor networks and ad hoc networks are:

a) The nodes in WSNs will be of very large number compared to other networks.

b) Sensor nodes will be densely deployed.

c) Due to harshness of the environment and energy depletion, sensor nodes are susceptible to catastrophes.

d) Topology changes will be usual in WSNs.

e) Sensor nodes usually adopt broadcast communication while point to point communications is adopted by ad hoc networks.

f) Sensor nodes face strict constraints in power, computational capacities and memory resource.

g) Because of the huge quantity of sensor nodes and the overheads, they usually may not have global identification.
Sensor nodes will be heavily deployed in majority of applications they are involved. As the sensor nodes are placed quite close by, subsequently multihop communication consumes less power and transmission power levels can be kept low, compared to traditional networks. The effects of signal propagation experienced in wide-ranging communication can also be minimised. But because of the constraints in energy resources, WSNs has to focus more on energy conservation techniques.

\subsection{Energy Efficient Techniques in WSN}

In WSN, sensors dissipate energy while sensing, processing, transmitting or receiving data to fulfill the mission required by the application. The other reasons for energy wastage are due to collision, overhearing, control packet overhead, idle listening and interference. There are five main classes of energy efficient techniques, namely, data reduction, protocol overhead reduction, energy efficient routing, duty cycling and topology control.

a) Data Reduction: It focuses on reducing the amount of data produced, processed and transmitted. In order to reduce the data in production, sampling and prediction based techniques are proposed. In processing and transmission, the data is reduced by means of data compression and data aggregation.

b) Protocol overhead reduction: This is used to increase the protocol efficiency by reducing the overhead. The overhead can be reduced by using adaptive transmission period, cross-layering and optimized flooding to avoid retransmission.

c) Energy efficient routing: Routing protocols should 
be designed with target of maximizing the network lifetime and minimizing the energy consumed by the end-to-end transmission. Some of the prominent routing approaches include opportunistic, data centric, hierarchical, geographical, multipath routing etc.

d) Duty cycling: It is the fraction of time the nodes are active during their lifetime. Duty cycling based on high granularity focus on selecting active nodes among all sensors deployed in the network. Duty cycling based on low granularity deals with switching off the radio of active nodes when no communication is required.

e) Topology control: It focuses on reducing energy consumption by adjusting the transmission power while maintaining network connectivity. This research focuses on maximizing the network lifetime by using the energy efficient techniques based on routing and data aggregation.

\subsection{Low Energy Adaptive Cluster Hierarchy Protocol}

W.R.Heinzelman, proposed a hierarchical clustering algorithm for wireless sensor networks, called Low Energy Adaptive Cluster Hierarchy protocol (LEACH) which is one of the popular hierarchical routing algorithm. The plan is to group of the sensor nodes based on the accepted signal force and use local group heads (CHs) as routers to the sink. This will accumulate energy because the transmissions will only be done by $\mathrm{CHs}$ instead of all sensor nodes. Optimal number of $\mathrm{CHs}$ is probable to be $5 \%$ of the total number of nodes. All the processing of data such as data union and aggregation are local to the cluster. CHs modify randomly over time for balancing the energy dissipation of nodes. There are various key features of LEACH are:

a) Coordinated locally and manage for cluster set-up and operation.

b) Cluster "base stations" or" Cluster-heads" rotated randomly and the Corresponding clusters.

c) Local firmness to reduce global communication.

In $\mathrm{LEACH}$, the procedure is separated into fixed-length rounds, everywhere each round starts with a setup phase after that a steady-state phase. LEACH is a hierarchical protocol in which most nodes transmit to cluster heads, and the cluster heads aggregate and compress the data and forward it to the base station (sink). Each node uses a stochastic algorithm at each round to determine whether it will become a cluster head in this round. LEACH assumes that each node has a radio powerful enough to directly reach the base station or the nearest cluster head, but that using this radio at full power all the time would waste energy.

Nodes that have been cluster heads cannot become cluster heads again for $P$ rounds, where $P$ is the desired percentage of cluster heads. Thereafter, each node has a $1 / P$ probability of becoming a cluster head in each round. At the end of each round, each node that is not a cluster head selects the closest cluster head and joins that cluster. The cluster head then creates a schedule for each node in its cluster to transmit its data.

All nodes that are not cluster heads only communicate with the cluster head in a TDMA fashion, according to the schedule created by the cluster head. They do so using the minimum energy needed to reach the cluster head, and only need to keep their radios on during their time slot.

\section{RELATED WORK}

(T. A. H. Hassan, G. Selim and R. Sadek, 2015) In Wireless Sensor Networks (WSN) with a clustered hierarchical structure, Cluster-Head $(\mathrm{CH})$ nodes are considered the interface between the leaf normal sensors and the Base Station (BS). The energy dissipation of the sensors, whatever their type, can be optimized by a load balancing in the packet TX/RX process in order to prolong the network lifetime and minimize the advertisement phase time for cluster head selection in each round of the LEACH-C protocol. This paper proposes a routing protocol for $\mathrm{LEACH}$ _C WSN in which the system lifetime can be extended by assigning a vice cluster head (VCH) to each of the CHs. Unlike other different VCH based protocols, the assigned $\mathrm{VCH}$ doest not remain in the idle state and receive its responsibility by the death of the $\mathrm{CH}$, rather the VCH shares the TX/RX load with its $\mathrm{CH}$ in order to balance the load distribution, shortly after the death of the $\mathrm{CH}$ a VCH is fully loaded until a new VCH receives the TX load. The simulation results confirm that the theoretically expected results. The functionality of the proposed protocol is tested under different simulation conditions such as the size of the WSN field and the results of simulation prove that the proposed protocol prolongs the network lifetime as expected.

(K. A. Darabkh, W. S. Al-Rawashdeh, M. Hawa, R. Saifan and A. F. Khalifeh, 2017) There are many existing clustering protocols that aim at making the sensor network stay functioning longer out of which Low-Energy Adaptive Clustering Hierarchy (LEACH) and Threshold-based LEACH (T-LEACH) protocols. T-LEACH protocol takes advantage of LEACH main deficiency, which is about having high control overhead. In other words, T-LEACH proposes that cluster heads do not have to turn over every round but rather every batch of rounds. Nodes will keep serving as cluster heads as long as their energy is higher than a threshold energy. This article imposes upon major drawbacks of TLEACH and proposes a Modified Threshold-based Cluster Head Replacement (MT-CHR). In MT-CHR, a new probability of being a cluster head, for any node in any round, has been proposed which agrees fairly with the assumptions introduced in LEACH protocol.

(K. Roshan and K. R. Sharma, 2018) The wireless sensor network is the decentralized type of network in which sensor nodes sense information and transmit to base station. The energy consumption is the major issue of wireless sensor network due to small size of sensor nodes and far deployment of the network. In this research paper, the Improved LEACH protocol is further improved by deploying cache nodes in the 
network. In the Improved LEACH protocol with cache nodes, the cluster head are selected on the basis of distance and energy. The cluster heads transmit data to cache node which is nearest and has minimum access time. The simulation of proposed modal is done in MATLAB. To validate the results of Improved LEACH protocol is compared with Improved LEACH protocol with cache node.

(T. Yang, Y. Guo, J. Dong and M. Xia, 2018) Aiming at the problem of short life cycle and uneven energy consumption of low power adaptive clustering hierarchical protocol (LEACH), an improved LEACH algorithm is proposed. In the selection of cluster head nodes, the optimal cluster head proportion is calculated by considering the problem of residual energy and current position, and the SEP algorithm is used to calculate the different cluster head election probability for the advanced nodes and ordinary nodes, so that the distribution of the cluster heads is more uniform. At the same time, in the data transmission phase, data communication is performed using a hybrid routing method. That is, the distance from the node to the cluster head and the base station is compared. When the node is close to the base station, the node directly communicates with the base station. Otherwise, it communicates with the base station through the cluster head. It reduces the number of clustering and saves network energy consumption. Simulation experiments show that the improved LEACH algorithm makes the distribution of dead nodes more discrete, the entire network energy consumption more balanced and extends the life cycle of the wireless sensor network.

(L. Mao and Y. Zhang, 2017) With limited battery power of nodes, communication energy consumption is the main factor to affect the lifetime of wireless sensor networks. It is of great significance to design a communication protocol to prolong the lifetime of the networks. In this paper, an energy-efficient LEACH algorithm for wireless sensor networks is proposed, which takes the energy and position factors of each node into account to optimize the cluster head election and data transmission mode. This method introduces the current energy and position factors of the node into the threshold to further reduce the randomness of the cluster head's designation. In the data transmission stage, some of the neighboring cluster heads are dedicated to be selected as the relay nodes to make the communication energy consumption balanced. The simulation results show that the proposed algorithm has higher stability and longer lifetime of the network compared to LEACH and LEACH-C algorithms.

(S. Soro, W.B. Heinzelman, 2005) This paper has suggested an unequal classification-size (UCS) model for network organizing that can lead to a more uniform energy dissipation between the main nodes of the cluster, thus increasing network life. We also extend this approach to homogenous sensor networks and show that UCS can also result in a more homogenous network with a uniform energy discharge .

(F. Bagci, 2016) This paper suggested the wireless sensor networks Energy Saving Token Ring Protocol (ESTR) to introduce an Energy Efficient Medium Access Control (EEMAC) protocol. ESTR is based on the popular fair and high-performance token ring protocol. The sensor nodes are linked to each other and form a ring that only triggers and communicates the node containing the token. ESTR uses this function for inactive sensor nodes that do not wish to send or receive messages for sleep cycles. Since the token holding times for the network are set, each time period can be determined by sensors. This results in much better energy outcomes and extends the life of the network as a whole. In addition, ESTR minimizes carbon loss by listening idle and listening. Moreover, several interconnected rings are endorsed in the proposed protocol. This decreases the size of the network and enables smaller rings to be formed based on geographical position. Connectivity in the network is maintained by the presence of many rings. The ESTR provides a versatile mechanism that allows the full ring size regulation and power consumption. In addition, energy information of the previous node is part of the token that is shared among the nearest nodes. For the next time, the present node takes its sleep to balance overall ring capacity. This dramatically enhances network life. The NS-2 network simulator is used to test the ESTR, and achieves the best energy outcomes in comparison with the others.

\section{PROPOSED WORK}

Without the need for a routing protocol, nodes within each transmission range can communicate. However, nodes which are not within each other's range, like nodes $A$ and $C$ in Figure 1, must send data to a middle node, for example B in Figure 4.1, which sends packet forward and reverse, because it overlaps the transmission ranges of both nodes $\mathrm{A}$ and $\mathrm{C}$.

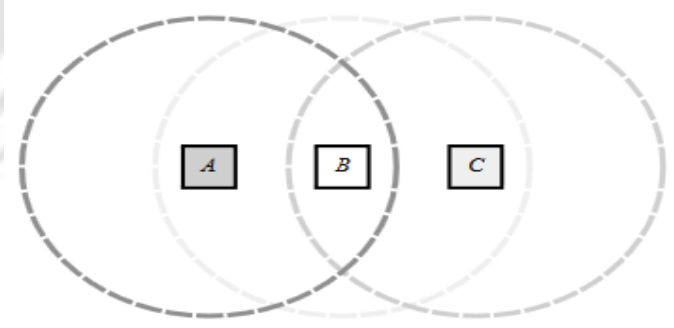

Figure 1 Number of Nodes with Ranges in a WSN

There may be direct contact between nodes when: (1) the nodes are neighbours and (2) the nodes are powerful enough. However, because of the large volume of energy required to achieve a high power transmission, this could be an inconvenience. It is possible to categorise routing protocols into:

(1) Flat protocols in which no master nodes or reference nodes have been found

(2) Hierarchy protocols in which certain nodes are given higher functions than others.

Figure 2 displays a WSN hierarchical cluster-based model. This network consists of several clusters, also known as the clumps, each of which is made up of a cluster head $(\mathrm{CH})$ that is responsible for data. Cluster members (CMs) are knots which are not cluster heads. $\mathrm{CHs}$ are responsible for intercluster and intercluster coordination. By coordination 
within the Cluster we mean coordination between nodes and aggregating their data within the same cluster. In contrast, the communication between $\mathrm{CHs}$ or between $\mathrm{CH}$ and $\mathrm{BSs}$ is intercluster coordination. CHs communicate between $\mathrm{CM}$, in other words, and between $\mathrm{CHs}$, BSs communicate.

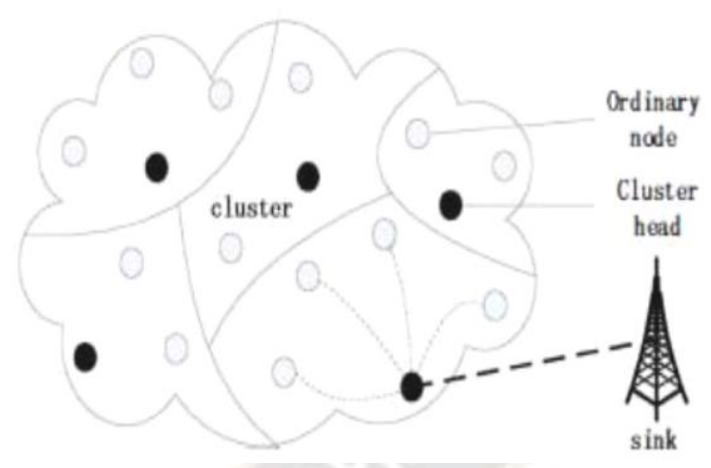

Figure 2 Cluster-Based Hierarchical Model

The number of CHs affects the consumption of energy. If a WSN contains many $\mathrm{CHs}$, energy consumption will increase through communication between the $\mathrm{CHs}$ and the BS. In addition, if the WSN contains a small number of $\mathrm{CHs}$, data aggregation and communication between $\mathrm{CMs}$ and $\mathrm{CH}$ will increase energy consumption. The most basic hierarchical protocol (the clusters-based protocol), which is the base for the majority of existing energy efficient protocols, is the Low Energy Adaptive Clusters (LEACH) Hierarchy. Figure 2 shows the LEACH clustering.

A set-up phase and a steady-state phase is completed by LEACH in several rounds. LEACH has two phases each:

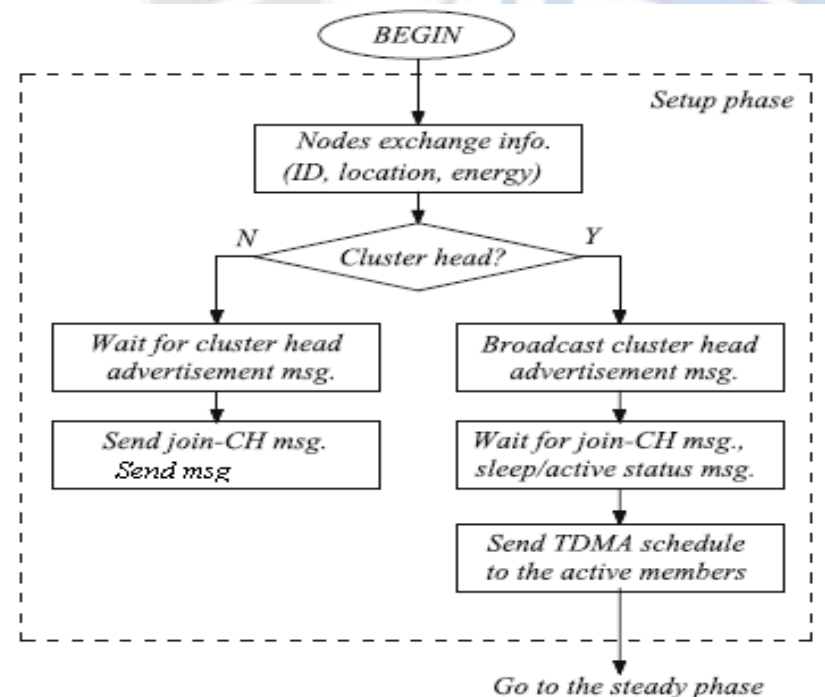

Figure 3 Set-up Phase

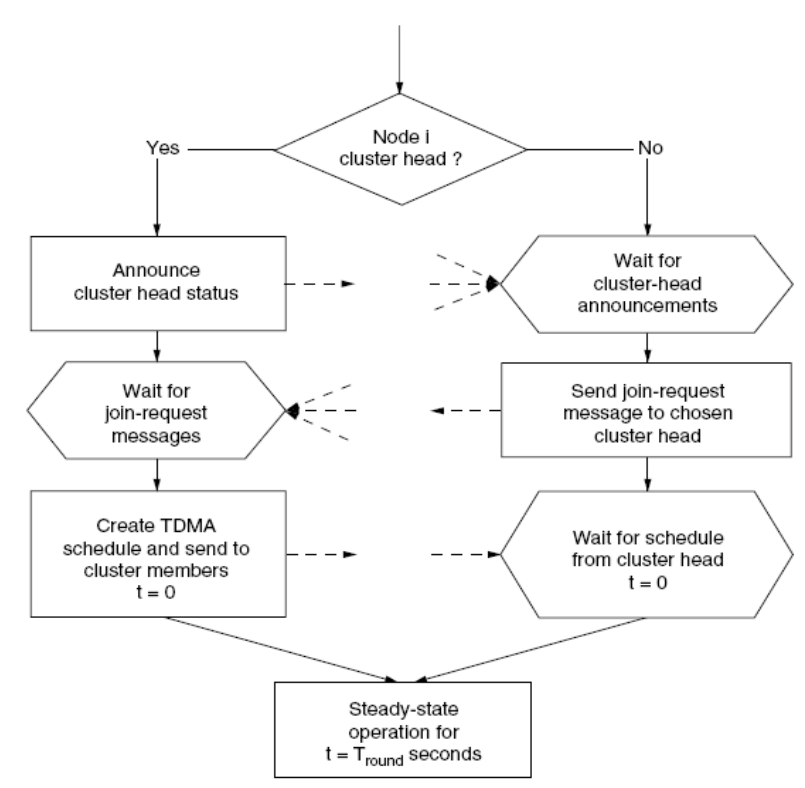

Figure 4 Steady Phase

Only the various parts that make up the communication process are depicted in Figure 5. In the model sensor node communicates using radio, and thus includes a radio component and a radio antenna. As a result, the component is further divided into receiver, transmitter, and amplifier to show the amount of energy it consumes.

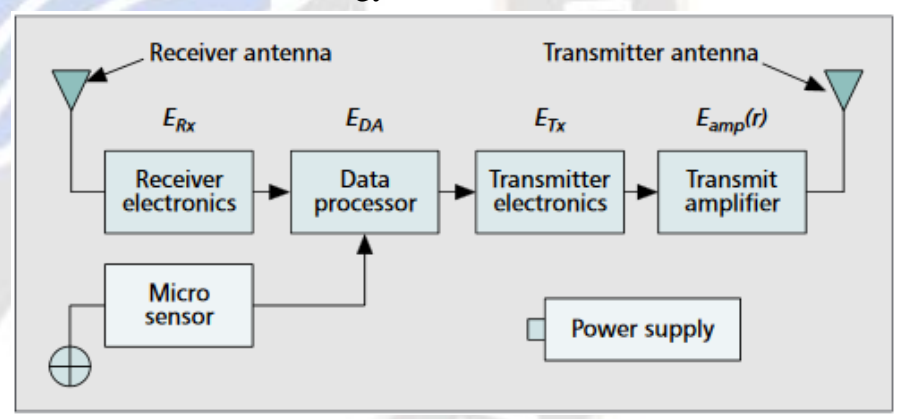

Figure 5 Energy Model

\section{RESULT ANALYSIS}

The results below display the simulation of both the LEACH and the LEACH-C protocols at 200 nodes and area of 400 $\mathrm{m}^{2}$

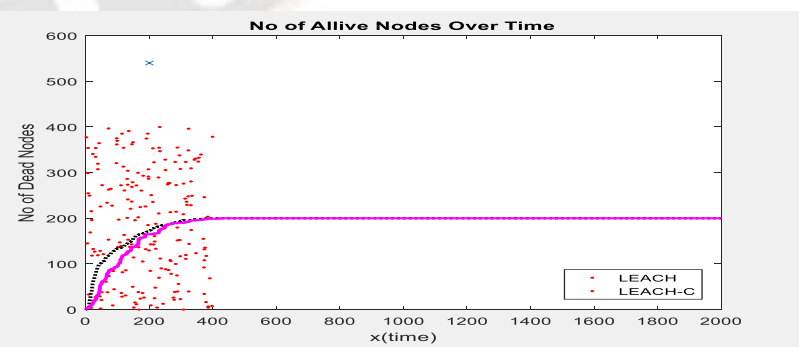

Figure 6 Number of Alive Nodes (LEACH-C)

The graph displayed in figure 6 shows the plot of dead nodes with respect to round number for LEACH-C protocol. It can be observed from the graph that the performance of proposed algorithm is superior to that of LEACH protocol. 
The graph displayed in figure 7 shows the number of packets send to sink node with respect to both protocol.

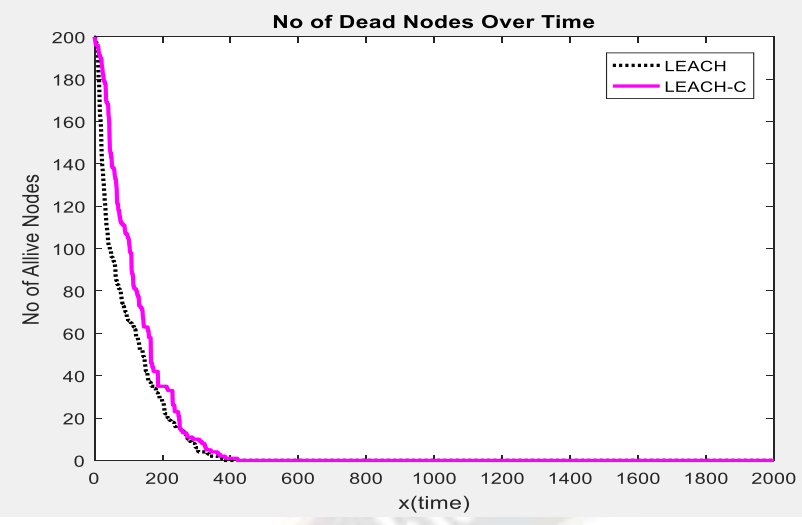

Figure 7 Dead Nodes

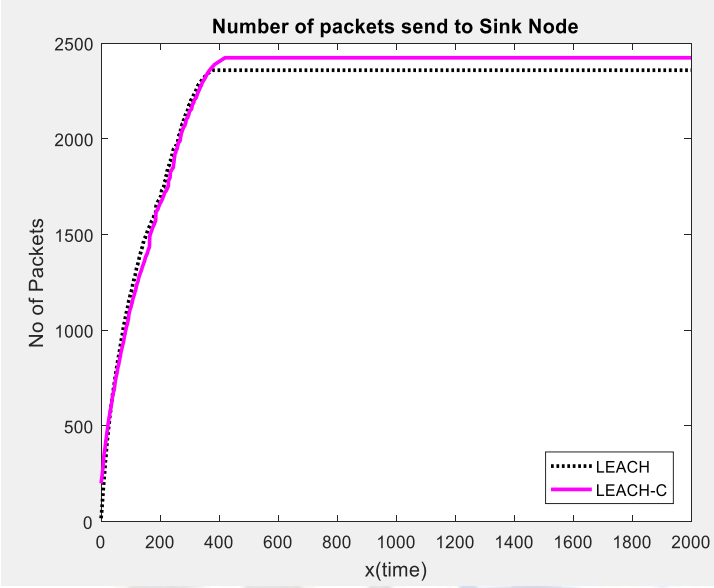

Figure 8 Number of Packets Send to Sink Node

The result displayed in figure 8 describes the scenario of number of dead nodes at with respect to number of rounds.

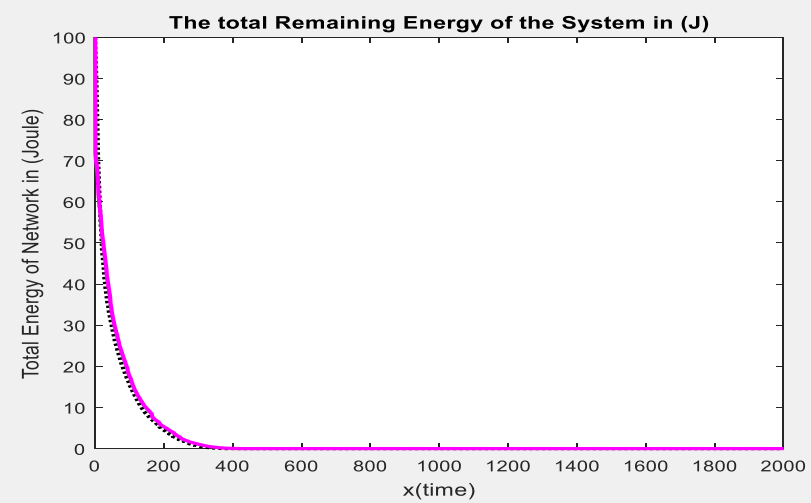

Figure 9 No. of Dead Nodes v/s Round No. (LEACH)

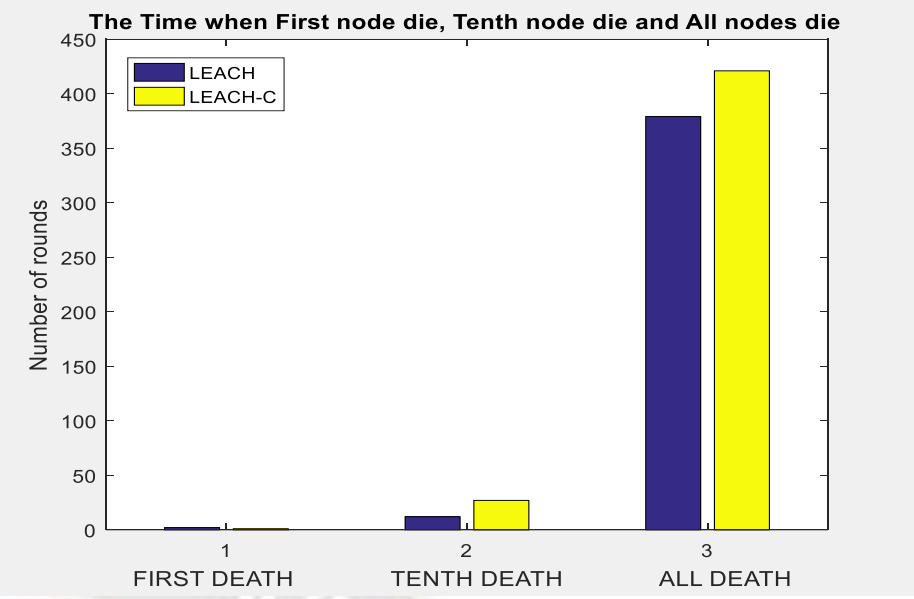

Figure 10 Node Death Analysis

The result displayed in figure 10 describes the scenario of number of dead nodes with respect to first death, tenth death and all death with respect to rounds. This is because the nodes or clusters that are more far from the base station are forced to dissipate large amounts of energy to send data, because they must move longer distances from those that are nearer. This is because LEACH-C is an inter-cluster routing device, which makes the network survive longer, than LEACH in most cases. LEACH-C is the only thing it does. However, LEACH communicates directly with the cluster head and then with the base station. While the company uses multi-hop systems, LEACH-C can achieve much better energy efficiency than LEACH with the use of multi-way and hierarchical routing parameters and techniques with the use of a multi-hop system.

\section{CONCLUSION}

In many cases, wireless sensor networks are usually dispersed across broad areas. There is a requirement in this respect for methods that can better manage the WSN. The limited battery capacity is used for wireless sensor networks. The key challenge in designing Wireless Sensor Network protocols is energy efficiency as the sensor nodes are restricted in capacity. The last motivation behind every routing protocol is to make the network work for a longer period of time as energy-efficient as possible. In this work, we introduced clustering as a means of overcoming this energy efficiency problem. Detailed description on the process of LEACH and LEACH-C two protocols is available. From the short analysis of the simulation, we concluded that LEACH can be used in smaller grids with less than 50 nodes in total, when it is somewhat better than LEACH-C and LEACH, in larger grids and when the heuristic probability of selecting Cluster Head is higher.

\section{REFERENCES}

[1] Zhen Zhao, Guangming Li, Menghui Xu, "An Improved Algorithm Based on LEACH Routing Protocol”, IEEE 19th International Conference on Communication Technology, 2019. 
[2] L. B. Bhajantri and A. V. Sutagundar, "Fuzzy logic based cluster head selection and data processing in Distributed Sensor Networks," in Proc. of 2016 International Conference on Computing, Analytics and Security Trends (CAST), Pune, Dec. 19-21,2016, pp. 282-288.

[3] A. Nandi, B. Sonowal, D. Rabha and A. Vaibhav, "Centered Sink LEACH Protocol for Enhanced Performance of Wireless Sensor Network,"in Proc. of 2019 International Conference on Automation, Computational and Technology Management (ICACTM), London, United Kingdom, Apr. 24-26,2019, pp. 436-440.

[4] H. Patel and V. Shah, "A review on energy consumption and conservation techniques for sensor node in WSN," in Proc. of 2016 International Conference on Signal Processing, Communication, Power and Embedded System (SCOPES), Paralakhemundi, Oct.3- 5,2016, pp. 594-599.

[5] M. N. Jambli, M. I. Bandan, K. S. Pillay and S. M. Suhaili, "An Analytical Study of LEACH Routing Protocol for Wireless Sensor Network," in Proc. of 2018 IEEE Conference on Wireless Sensors (ICWiSe), Langkawi, Malaysia, Nov.2122,2018, pp. 44-49.

[6] D. M. Birajdar and S. S. Solapure, "LEACH: An energy efficient routing protocol using Omnet++ for Wireless Sensor Network," in Proc. of 2017 International Conference on Inventive Communication and Computational Technologies (ICICCT), Coimbatore, Mar.10-11, 2017, pp. 465-470.

[7] T. A. H. Hassan, G. Selim and R. Sadek, "A novel energy efficient vice Cluster Head routing protocol in Wireless Sensor Networks," in Proc. of 2015 IEEE Seventh International Conference on Intelligent Computing and Information Systems (ICICIS), Cairo, Dec.12- 14,2015, pp. 313-320.

[8] K. A. Darabkh, W. S. Al-Rawashdeh, M. Hawa, R. Saifan and A. F. Khalifeh, "A novel clustering protocol for wireless sensor networks," in Proc. of 2017 International Conference on Wireless Communications, Signal Processing and Networking (WiSPNET), Chennai, Mar.22-24,2017, pp. 435438.

[9] K. Roshan and K. R. Sharma, "Improved LEACH Protocol with Cache Nodes to Increase Lifetime of Wireless Sensor Networks," in Proc. of 2018 2nd International Conference on Trends in Electronics and Informatics (ICOEI), Tirunelveli, May.11-12,2018, pp. 903-908.

[10] T. Yang, Y. Guo, J. Dong and M. Xia, "Wireless Routing Clustering Protocol Based on Improved LEACH Algorithm," in Proc. of 2018 IEEE International Conference on RFID Technology \& Application (RFID-TA), Macau, Sept.26-28, 2018, pp. 1-6.

[11] L. Mao and Y. Zhang, "An energy-efficient LEACH algorithm for wireless sensor networks," in Proc. of 2017 36th Chinese Control Conference (CCC), Dalian, July.26-28,2017, pp. 9005-9009.

[12] S. Soro, W.B. Heinzelman, Prolonging the lifetime of wireless sensor networks via unequal clustering. IEEE International Parallel \& Distributed Processing Symposium 365 (2005)

[13] F. Bagci, Energy-efficient communication protocol for wireless sensor networks. Ad Hoc \& Sensor Wireless Networks 30(3-4), 301-322 (2016).

[14] Y. Zhai, L. Xu, Ant colony algorithm research based on pheromone update strategy, 20157 th Int. Conf. Intell. Human-Machine Syst. Cybern. 1(2), 38-41 (2015).

[15] W. Ding, W. Fang, Target tracking by sequential random draft particle swarm optimization algorithm. IEEE Int Smart Cities Conf. 2018, 1-7 (2018).
[16] N. Thi, H. Thi, T. Binh, N. Xuan, An efficient genetic algorithm for maximizing area coverage in wireless sensor networks. Inf. Sci. (Ny). 488, 58-75 (2019).

[17] M. Ben Salah, A. Boulouz, "Energy efficient clustering based on LEACH", IEEE International Conference on Engineering \& MIS (ICEMIS), 2016.

[18] Alka Singh, Shubhangi Rathkanthiwar, Sandeep Kakde, "LEACH based-energy efficient routing protocol for wireless sensor networks", IEEE International Conference on Electrical, Electronics, and Optimization Techniques (ICEEOT, 2016.

[19] S. Al-sodairi, R. Ouni, Sustainable computing: informatics and systems reliable and energy-efficient multi-hop LEACHbased clustering protocol for wireless sensor networks. Sustain. Comput. Informatics Syst. 20, 1-13 (2018).

[20] G.K. Nigam, C. Dabas, ESO-LEACH: PSO based energy efficient clustering in LEACH. J. King Saud Univ. - Comput. Inf. Sci. 1, 4-11 (2018).

[21] N. Mazumder, H. Om, Distribute fuzzy approach to unequal clustering and routing algorithm for wireless sensor networks. International Journal of Communication Systems 31(12) (2018).

[22] N.G. Palan, B.V. Barbadekar, S. Patil, Low energy adaptive clustering hierarchy (LEACH) protocol: a retrospective analysis. International Conference on Inventive Systems and Control (ICISC), 363-374 (2017, 2017).

[23] K.A.Z. Ariffin, R.M. Mokhtar, A.H.A. Rahman, Performance analysis on LEACH protocol in wireless sensor network (WSN) under black hole attack. Advanced Science Letters 24(3), 1791-1794 (2018).

[24] Manish Gupta, Krishnanand K R, Hoang Due Chinh, Sanjib Kumar Panda, "Outlier detection and data filtering for wireless sensor and actuator networks in building environment”, IEEE ICBEST, Singapore, pp 95-100, 2015.

[25] Mohamed Younis, Izzet F. Senturk, Kemal Akkaya, Sookyoung Lee, Fatih Senel, "Topology management techniques for tolerating node failures in wireless sensor networks: A survey", Computer Networks Elsevier Vol.No. 58, pp 254-283, 2014.

[26] Suat Ozdemir and Yang Xiao, "Outlier detection-based faulttolerant data aggregation for wireless sensor networks", Security and Communication Networks, John Wiley \& Sons, Ltd., pp 702-710, 2013.

[27] Mauro Migliardi, Alessio Merlo, and Luca Caviglione, "A survey of Green, Energy-Aware Security and Some of its Recent Developments in Networking and Mobile Computing", in 2014 Eighth International Conference on Innovative Mobile and Internet Services in Ubiquitous Computing (IMIS), Vol.No.24, pp 241-246, July 2014.

[28] Tarachand Amgoth, Prasanta K Jana, "Energy-aware Routing algorithm for Wireless sensor networks", Elsevier Computers and Electrical Engineering, pp 357-367, 2015.

[29] Alessio Merlo, Mauro Migliardi, Luca Caviglione, "A survey on energy-aware security mechanisms", Pervasive and Mobile Computing, Vol. 24, pp 77-90, December 2015. 